\title{
Potential role of artificial intelligence in craniofacial surgery
}

\author{
Jeong Yeop Ryu ${ }^{1}$, \\ Ho Yun Chung ${ }^{1,2}$, \\ Kang Young $\mathrm{Choi}^{1}$ \\ ${ }^{1}$ Department of Plastic and \\ Reconstructive Surgery and ${ }^{2}$ Cell \& \\ Matrix Research Institute, School of \\ Medicine, Kyungpook National \\ University, Daegu, Korea
}

\begin{abstract}
The field of artificial intelligence (Al) is rapidly advancing, and Al models are increasingly applied in the medical field, especially in medical imaging, pathology, natural language processing, and biosignal analysis. On the basis of these advances, telemedicine, which allows people to receive medical services outside of hospitals or clinics, is also developing in many countries. The mechanisms of deep learning used in medical Al include convolutional neural networks, residual neural networks, and generative adversarial networks. Herein, we investigate the possibility of using these Al methods in the field of craniofacial surgery, with potential applications including craniofacial trauma, congenital anomalies, and cosmetic surgery.
\end{abstract}

Abbreviations: Al, artificial intelligence; ANN, artificial neural network; CNN, convolutional neural network; CT, computed tomography; DNN, deep neural network; FDA, Food and Drug Administration; GAN, generative adversarial network; MR, magnetic resonance; NIRS, near-infrared spectroscopy; POD, postoperative day; RNN, recurrent neural network.

Keywords: Artificial intelligence / Neural networks, computer / Surgery, plastic

\section{INTRODUCTION}

Many new technologies, such as artificial intelligence (AI), three-dimensional printing, virtual and augmented reality, and robotic surgery, are being introduced to the medical field. Large amounts of data are available in the specialty of craniofacial surgery, including photographs, computed tomography (CT) images, and audio files. These data provide a basis for applying AI technology. To date, the most prominent application of AI in medicine is image recognition technology, as exemplified by AI models used in radiology and pathology. The applications of AI include image quality improvement and the identification, measurement, and classification of lesions. The performance of speech recognition and natural language processing has also

Correspondence: Kang Young Choi

Department of Plastic and Reconstructive Surgery, School of Medicine,

Kyungpook National University, 680 Gukchaebosang-ro, Jung-gu, Daegu 41944,

Korea

E-mail: kychoi@knu.ac.kr

Received October 7, 2021 / Revised October 20, 2021 / Accepted October 20, 2021 substantially improved.

The US Food and Drug Administration (FDA) has approved several medical AI applications in fields including cardiology, endocrinology, radiology, neurology, internal medicine, ophthalmology, emergency medicine, and oncology; however, no AI models developed for plastic and reconstructive surgery have yet received FDA approval [1]. Nonetheless, medical AI holds considerable promise across the full range of medical specialties, including plastic surgery. In this review, we would like to investigate the potential of applying medical AI to plastic surgery, especially in the subspecialty of craniofacial surgery.

\section{DEFINITION OF AI}

To understand the definition of AI, it is essential to be familiar with the conceptual relationships among AI, machine learning, and deep learning (Fig. 1). AI is a term with a very broad meaning, extending beyond the field of engineering to include political, economic, and social considerations. Instead, machine 


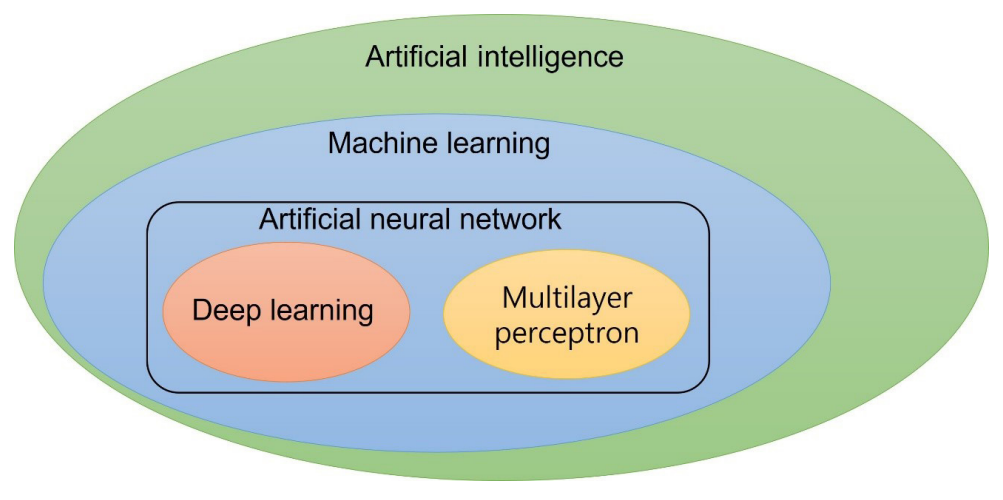

Fig. 1. Relationship of artificial intelligence, machine learning, and deep learning.

Neural network

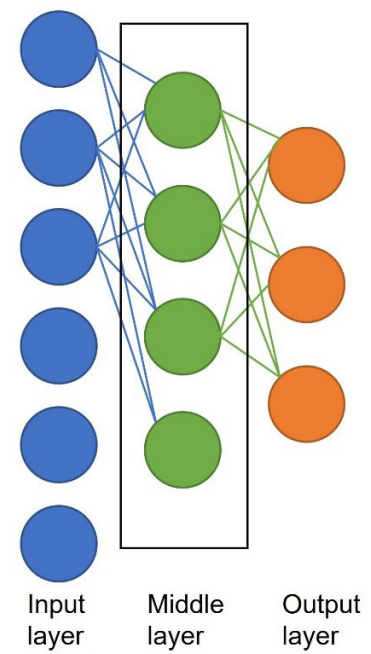

Deep learning

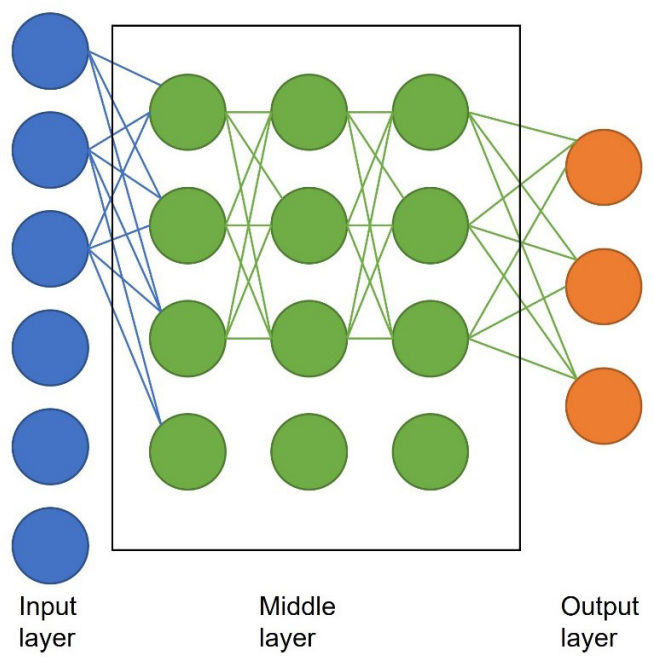

Fig. 2. Deep learning and a neural network. Deep learning neural networks are similar to biological neural networks.

learning refers to the implementation of AI limited to an engineering context. Machine learning encompasses various methods, including those based on artificial neural networks (ANNs). An ANN simulates human neurons and neural networks that involve hierarchical connections. The ANN concept was first proposed by McCulloch and Pitts [2] and made concrete by Rosenblatt [3], who suggested a structure, called a perceptron, that mimics the signal transduction process of human nerve cells. Hinton and Salakhutdinov [4] coined the term "deep neural network" (DNN) for a multilayer perceptron composed of several hidden layers and described the learning method of a DNN as "deep learning." Deep learning, which refers to an ANN consisting of several deep layers, incorporates most characteristics of machine learning (Fig. 2).

Medical applications based on deep learning include image recognition, classification, detection, and segmentation; face recognition; visual tracking; video classification; speech recognition; and natural language processing [5].

\section{CONVOLUTIONAL NEURAL NETWORKS AND CRANIOFACIAL SURGERY}

With advances in deep learning technology based on DNNs and the introduction of the convolutional neural network (CNN) technology, a paradigm shift has occurred in the machine learning field dealing with image data. Before the CNN concept was presented, LeCun et al. [6] tried to classify input features by introducing the convolution concept into the multilayer perceptron structure and presented a methodology to use this concept for the handwritten number recognition problem. A CNN is an algorithm used for image pattern recognition that makes it possible to carry out the entire process from feature extraction to classification using a single model (Fig. 3). CNNs can be applied as an essential structure for detection and segmentation problems beyond simple image classification; hence, they have become a vitally important technology in deep learn- 

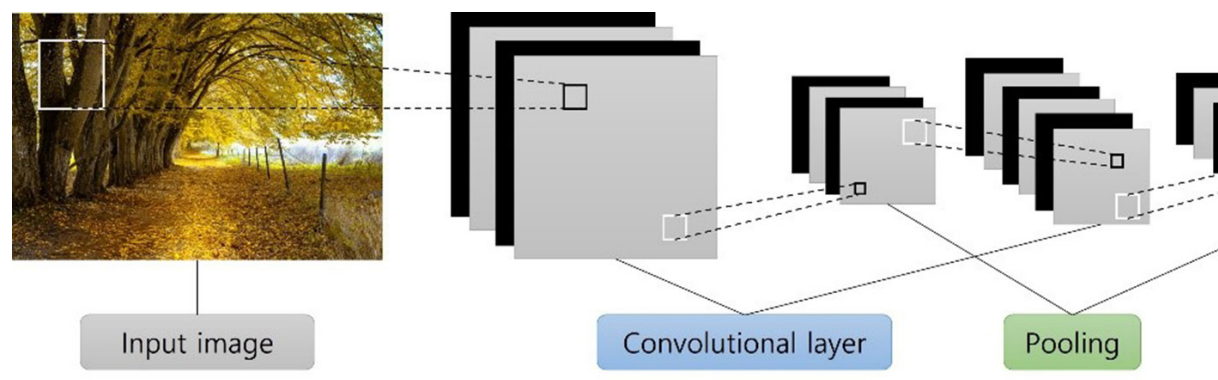

Feature extraction

Feature reduction

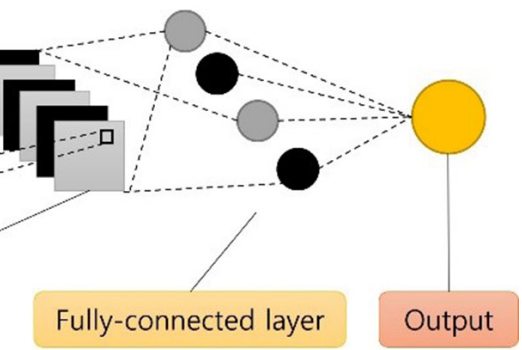

Final classification

Fig. 3. Basic structure of convolutional neural networks (CNNs). A CNN consists of three stages: feature extraction, feature reduction, and the final classification.

ing studies dealing with medical images [7]. In imaging research, classification refers to determining the presence or absence of a specific object in an image, while detection involves both checking for the presence or absence of a specific object in an image and determining the object's location. Segmentation refers to displaying the position of a specific object in an image in pixel units. Research in this domain aims to construct a CNN that derives an output image from an original image, showing a mask of the object of interest in the original input image.

Many potential applications in the field of craniofacial trauma are based on CNNs. Most craniofacial surgeons diagnose craniofacial trauma based on the patient's history, a physical examination, and imaging findings, such as $\mathrm{CT}$ and radiography $[8,9]$. CT scans are the most important diagnostic tool for diagnosing craniofacial trauma. AI can automatically diagnose craniofacial bone fractures using CNN-based classification or detection mechanisms. The effectiveness of CNN-based imaging diagnostics for bone fractures in the extremity, spine, and hip has already been reported in several studies [10-19]. In these studies, the pretrained CNN models used were ResNet-152, DenseNet, Inception v3, U-Net, VGG_16, VGG_19, Networkin-Network, VGG CNN's Network, and BVLC Reference CaffeNet. The mean accuracy was $90.08 \%$ (range, $83 \%-98 \%$ ), and the mean area under the curve was 0.98 (range, 0.95-1.00). Classification, detection, and segmentation using CNN models can be used for patients with facial bone fractures based on Xray or facial CT images.

Interestingly, several reports have compared the medical image reading performance of AI with that of doctors. In fundus photographs from adults with diabetes, an algorithm based on deep machine learning showed high sensitivity and specificity for detecting referrable diabetic retinopathy. The performance of this algorithm was on par with the diagnostic accuracy of ophthalmologists [20]. For classifying important dermatological diseases in general skin photos or dermoscopy photos, an
AI algorithm showed a similar level of accuracy to that of experienced dermatologists [21]. The performance of an AI algorithm in finding malignant pulmonary nodules in chest radiography images exceeded the diagnostic accuracy of experienced chest radiologists [22]. In addition, several studies have reported that the image reading performance of AI algorithms was superior or similar to that of doctors $[23,24]$. Based on these studies, if CT or X-ray images of patients with facial bone fractures are trained well enough, it may be possible to implement an AI algorithm with similar image reading performance to that of craniofacial surgeons.

With regard to orthognathic surgery, AI algorithms can be applied to maxillofacial imagery, treatment planning, custom orthodontics, surgical appliances, and treatment follow-up. Software using AI to perform automatic tracing through deep learning of cephalograms already exists [25]. At present, attempts are being made to use machine learning to perform surgical planning and automatically produce CAM/CAD surgical appliances.

CNNs can also be applied to vascular diseases in the craniofacial field. Several studies have reported that AI algorithms showed excellent performance in automatic segmentation of areas affected by intracranial hemorrhage using brain CT images and in measurements of hemorrhage volume [26-29]. The possibility of accurate automatic segmentation of the extent and volume of vascular tumors or malformations can be considered, even for vascular anomalies in the head and neck area [30].

\section{RECURRENT NEURAL NETWORKS AND SPEECH AND NATURAL LANGUAGE PROCESSING: CLEFT PALATE AND VELOPHARYNGEAL INSUFFICIENCY}

One might imagine an $\mathrm{AI}$ algorithm listening to speech input 
from a smartphone and delivering a quantitative estimate of improvements in articulation. With recent advances in AI for voice recognition, this fantasy is becoming a reality. Language input constitutes representative "time series" data. In this data structure, a significant amount of data is not acquired at a single moment, as is the case for medical images; instead, data are continuously acquired in a sequence over time. Therefore, the data acquired according to a time sequence possess the characteristic of having an order, which is why the term "time series" data is used. It is difficult to reflect the time series characteristics of data in training with the aforementioned machine learning algorithms or ANN structures. Therefore, a machine learning algorithm that can effectively learn time series data has been proposed. The recurrent neural network (RNN) structure has been proposed as a representative algorithm for this purpose, and various modifications have been developed and are currently widely applied in both medical and nonmedical fields. The general process of a DNN is that when one datum is input at a time, the value of that datum is transmitted only in the direction of the output layer through an operation in each layer. Operations on nodes in each layer are performed using only the values received from the previous layer. Contrary to this, in an RNN, one datum is not input only once; instead, values in units of time are sequentially input. To express the correlation between the values input at each point in time, the output value of the node is transmitted as an input to the node of the next layer. At the same time, it is copied and returned to the input of its own node at the next point in time (Fig. 4). The recurrent edge-defined as the path through which data values that are sequentially entered from nodes in the hidden layer return-is the most specific feature of RNNs.

Mundt et al. [31] analyzed the voices of patients with depression using a statistical regression model. The results suggested the possibility that voice could serve as a biomarker indicating the degree of response to treatment. Benba et al. [32] reported the possibility of voice-based diagnosis based on an analysis of the voice characteristics of patients with Parkinson's disease through a machine learning algorithm. For children with cleft palate who have undergone palatoplasty, an algorithm could be developed using an RNN that would use spectral analysis to extract characteristic voice features from a picture pronunciation test or a sentence pronunciation test and extracting characteristic voice features. Cleft palate and velopharyngeal insufficiency are also closely related to speech $[33,34]$. Although currently not valid for children with cleft palate or velopharyngeal insufficiency, South Korea has a platform that uses AI algorithms to diagnose and conduct rehabilitation of speech disorders (https://www.talkytalky.kr/). This platform is currently only available in the Korean language. A different AI algorithm would be needed to expand the platform to include other languages (e.g., English, Japanese, Chinese, or Spanish).

\section{GENERATIVE ADVERSARIAL NETWORKS AND CRANIOFACIAL SURGERY}

Machine learning, which implements AI in software, refers to an algorithm in which a computer learns data, finds patterns on its own, and learns to perform appropriate tasks. Machine learning is classified as supervised, unsupervised, and reinforcement learning. The aforementioned CNNs and RNNs are supervised learning algorithms. A generative adversarial network (GAN) is a representative example of an unsupervised learning algorithm. The GAN is a regression model published by Ian Goodfellow that consists of a model responsible for classification (discriminator) and regression generation (generator) [35]. In these models, the generator and discriminator compete against each other to improve each other's performance. This is often compared to a confrontation between police and counterfeiters. The banknote counterfeiter (generator) tries as hard as possible to deceive the police (discriminator). Meanwhile, the police try to classify counterfeit and real banknotes. This com-

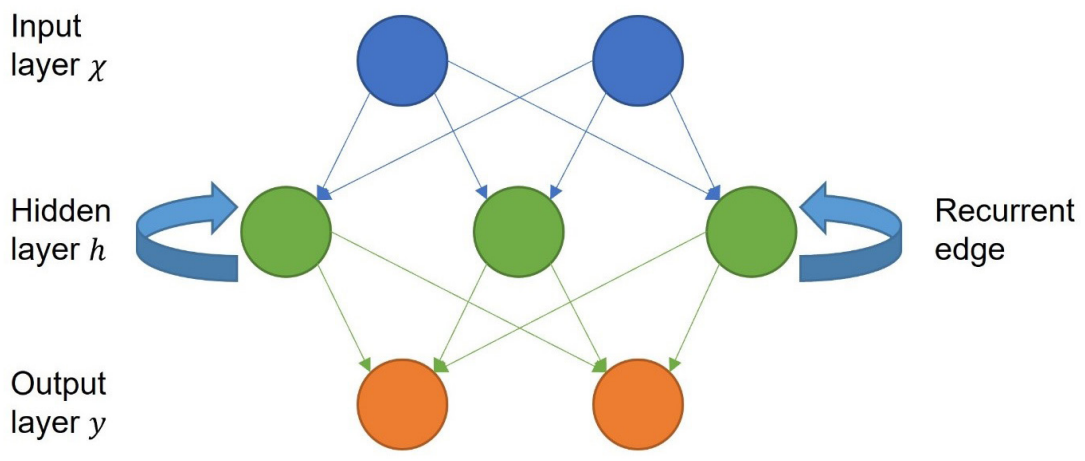

Fig. 4. Recurrent neural networks (RNNs). Unlike convolutional neural networks, RNNs have recurrent edges. 
petition involves continuous learning, so that the real and counterfeit bills become indistinguishable, resulting in the production of fake bills that are also virtually indistinguishable from the real ones. For instance, the generator receives an input of "Dog" and creates a certain image. The generator learns to deceive the discriminator, such that the discriminator can output it as a real image, denoted by 1 . In contrast, the discriminator alternately receives fake images created by the generator and real images that actually exist and learns to classify an image as 1 when it is real and 0 when it is fake (Fig. 5). The training of the generator and the discriminator occur alternately. The generator gradually develops into a model that can simulate the actual distribution well. The loss function of a GAN, including the meaning of this learning process, is defined as follows [35]:

$$
\min _{G} \max _{D} V(D, G)=E_{x \sim P \operatorname{data}(x)}[\log D(x)]+E_{z \sim P z(z)}[\log (1-D(G(z)))]
$$

$D(x)$ is a discriminator and a value between 0 and 1 ; thus, $D(x)$ yields a value of 1 if the datum is real and 0 if it is fake. The discriminator $D(G(z))$ has a value of 1 if the datum generated by the generator $\mathrm{G}, G(z)$, is judged as real and 0 if it is judged as fake. From the perspective of how the generator $G$ learns to minimize $V(D, G), \log (1-D(G(z)))$ must be minimized to make the second term of the above equation as small as possible. Therefore, $\log (1-D(G(z)))$ must be 0 , and $D(G(z))$ must be 1 . In other words, the generator must be trained to generate fake data that are perfect enough for the discriminator to classify as genuine. Many different types of GAN structures are being developed, including cycle GAN, conditional GAN, and progressive growing of GANs [36-38]. Among them, cycle GAN has a conversion structure that cycles the output of one domain to the input of another (Fig. 6).

Cycle GAN is being studied as a method to freely convert CT and magnetic resonance (MR) images in the medical field. In one study, CT images were generated from MR images using cycle GAN [39]. In the craniofacial surgery field, CT images could be generated using this algorithm. A recent large scale population-based cohort study found that CT scan exposure of young individuals between 0 and 19 years found that CT scan exposure was associated with an increased incidence of cancer

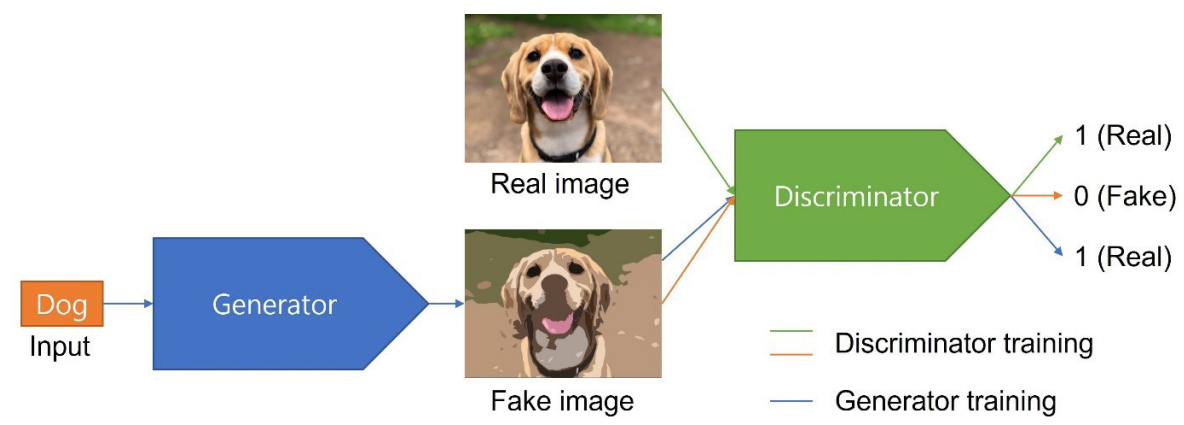

Fig. 5. Structure and learning method of generative adversarial networks (GANs). Two models appear in a GAN: a generator and a discriminator. The generator generates an image from an input. The generator learns to generate images that, when input to the discriminator, will be erroneously classified as real, as denoted by 1 . In contrast, the discriminator alternately receives fake images created by the generator and real images that actually exist and learns to classify an image as 1 if it is a real image or 0 if it is fake. The training of the generator and the discriminator occur alternately. The generator gradually develops into a model that can simulate the actual distribution well.

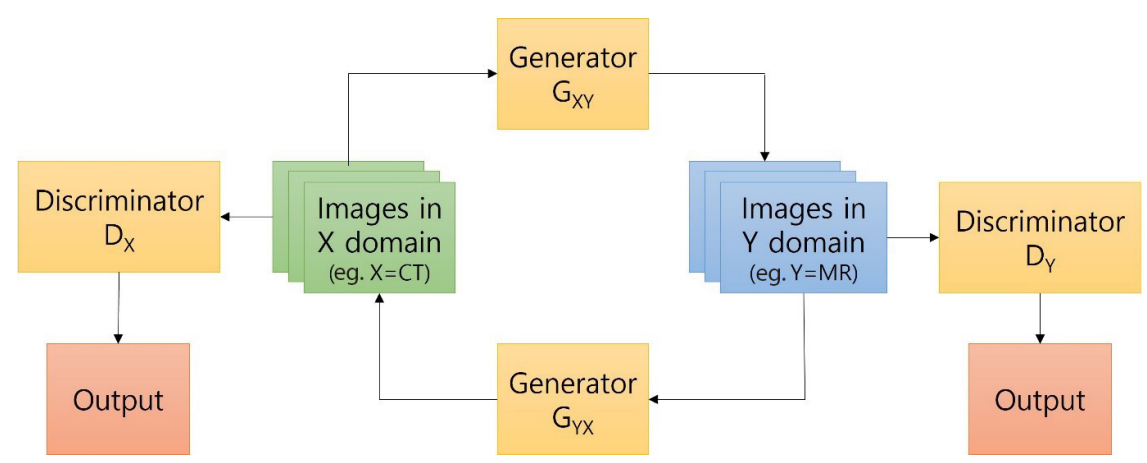

Fig. 6. Cycle generative adversarial network (GAN) structure. Cycle GAN trains a model that can convert between two domains of interest (X, $\mathrm{Y})$. It learns by calculating the cycle-consistency loss of images generated by $\mathrm{X} \rightarrow \mathrm{Y} \rightarrow \mathrm{X}$ or $\mathrm{Y} \rightarrow \mathrm{X} \rightarrow \mathrm{Y}$. CT, computed tomography; MR, magnetic resonance. 
in Koreans. The incidence of many types of lymphoid, hematopoietic, and solid cancers significantly increased after CT scan exposure. Among individuals who underwent CT scans, the overall cancer incidence was higher among exposed than nonexposed individuals after adjusting for age and sex (incidence rate ratio, 1.54; 95\% confidence interval, $1.45-1.63 ; p<0.001$ ) [40]. Despite high radiation risks in infants or children CT images are an essential tool for the diagnosis and treatment of patients with cleft lip with or without cleft palate, alveolar cleft, craniosynostosis, and pediatric facial bone fractures. In cases where a CT scan for infants or children would normally be required, it might instead be possible to take MR images and convert them into CT images using cycle GAN.

In aesthetic surgery, postoperative photographs can be created by inputting preoperative photos because GAN is an imagegenerating algorithm. In particular, postoperative photos of various results can be created specific to the surgical method. GANs can also generate voices, making it possible to generate a target voice for a child who needs speech therapy, such that the child can listen to the target voice for training purposes. In the craniofacial surgery field, the recently published style GAN showed the potential to create images with cosmetic deformities or congenital anomalies [41]. The images generated by GANs are not copyrighted; thus, they can be freely used in other studies.

At this point, GANs have the limitation of it being extremely difficult to control the attributes (e.g., gender, age, and hairstyle) of the image synthesized through the generator. Moreover, the generated image quality is inconsistent. In reality, unlike the results reported in previous articles, many unnatural images are created. Nonetheless, GANs still have the potential to be used in various ways, and we think that they have a greater possibility of being used in plastic surgery than in other medical fields.

\section{BIOSIGNAL DATA AND HEAD AND NECK RECONSTRUCTION}

Biomedical signals comprise observations of the physiological activities of organisms, ranging from gene and protein sequences to neural and cardiac rhythms [42]. With the development of monitoring techniques, such as electrocardiography and electroencephalography, a vast amount of data on biosignals can be collected, and AI algorithms can be applied to the collected data. AI research using biosignals is being actively conducted on models to predict a prognosis. One study reported an algorithm for screening hyperkalemia through deep learning of electrocardiograms. Another study reported an algorithm for predicting cardiac arrest in advance through deep learning
$[43,44]$.

In head and neck reconstruction, monitoring of free flaps is performed at 1-hour intervals on postoperative day (POD) 1 , at 2-hour intervals on POD 2, and at 4-hour intervals on POD 3-7 by visual examinations or using Doppler ultrasonography [45]. Reports of flap monitoring using near-infrared spectroscopy (NIRS) in the plastic surgery field have recently been presented [46-48]. The studies reported to date have described NIRS monitoring of flaps used for breast reconstruction. To the best of our knowledge, few reports have described flap monitoring using NIRS after head and neck reconstruction procedures; however, it is also possible to use NIRS to monitor free flaps used for head and neck reconstruction. An advantage of NIRS is that it can monitor muscle flaps with deep tissue (e.g., those utilized in facial palsy reconstruction); hence, it would be possible to use NIRS to monitor invisible and buried free flaps used for head and neck reconstruction. These NIRS data constitute biosignal data that can be collected in vast quantities. Accordingly, an algorithm that can predict flap failure in advance can be imagined. The authors are preparing an AI study related to flap monitoring. If we can create an algorithm that predicts flap failure in advance through deep learning, we could imagine scenarios in which surgeons receive notifications of impending flap failure, prompting them to prepare for readmission of the patient to the operating room.

\section{LIMITATIONS OF AI IN MEDICINE}

Although GAN is a type of unsupervised learning, many AI technologies are still based on supervised learning, which results in some limitations. First, for AI to perform a specific task automatically, a large amount of training data is required for technical instruction each time, and models must be individually developed using specific training data. In addition, the phenomenon of overfitting (i.e., excessive adaptation to the learning data) can occur, and when AI models are applied in realworld medical contexts, their performance is often poor. Therefore, the performance observed in the development stage is difficult to generalize to the actual medical field. Another important limitation is that $\mathrm{AI}$ algorithms that contain numerous variables cannot be intuitively understood by humans; thus, even if models achieve a certain level of performance, humans cannot fully understand the working principle. In the medical field, where algorithms must be applied to real people, it is difficult to embrace the use of algorithms with opaque operating principles that humans cannot comprehend. Finally, the performance of an AI system depends not only on the sophistication of its algorithm, but also on whether it learns a large amount of 
high-quality data. After ensuring patient safety and achieving a basic level of performance using high-quality data, the performance of a model should be improved by relearning using field data from multiple institutions.

\section{CONCLUSION}

Medical AI technology will ultimately play a key role in solving many of the problems facing healthcare. This will be possible through convergence and collaboration in various fields, including medicine, engineering, policy, and industry. Craniofacial surgeons do not need to understand and know every detail of AI technology at the level of engineers specializing in AI, but it is very important for them to understand its core characteristics and technical architecture and acquire knowledge on how to evaluate the performance and characteristics of developed technologies.

\section{NOTES}

\section{Conflict of interest}

Kang Young Choi is an editorial board member of the journal but was not involved in the peer reviewer selection, evaluation, or decision process of this article. No other potential conflicts of interest relevant to this article were reported.

\section{ORCID}

Jeong Yeop Ryu https://orcid.org/0000-0003-2812-5051

Ho Yun Chung https://orcid.org/0000-0001-7359-3044

Kang Young Choi https://orcid.org/0000-0002-7735-1939

\section{Author contribution}

Conceptualization: Jeong Yeop Ryu. Data curation: Jeong Yeop Ryu. Formal analysis: Jeong Yeop Ryu. Methodology: Jeong Yeop Ryu, Ho Yun Chung. Project administration: Jeong Yeop Ryu. Visualization: Jeong Yeop Ryu. Writing - original draft: Jeong Yeop Ryu. Writing - review \& editing: Kang Young Choi. Investigation: Jeong Yeop Ryu. Resources: Ho Yun Chung. Supervision: Kang Young Choi. Validation: Jeong Yeop Ryu. All authors read and approved the final manuscript.

\section{REFERENCES}

1. Benjamens S, Dhunnoo P, Mesko B. The state of artificial intelligence-based FDA-approved medical devices and algorithms: an online database. NPJ Digit Med 2020;3:118.

2. McCulloch WS, Pitts W. A logical calculus of the ideas immanent in nervous activity. 1943. Bull Math Biol 1990;52:99-115.
3. Rosenblatt F. The perceptron: a probabilistic model for information storage and organization in the brain. Psychol Rev 1958;65:386-408.

4. Hinton GE, Salakhutdinov RR. Reducing the dimensionality of data with neural networks. Science 2006;313:504-7.

5. Anwar SM, Majid M, Qayyum A, Awais M, Alnowami M, Khan MK. Medical image analysis using convolutional neural networks: a review. J Med Syst 2018;42:226.

6. LeCun Y, Bottou L, Bengio Y, Haffner P. Gradient-based learning applied to document recognition. Proceedings of the IEEE 1998;86:2278-324.

7. Yamashita R, Nishio M, Do R, Togashi K. Convolutional neural networks: an overview and application in radiology. Insights Imaging 2018;9:611-29.

8. Lee CL, Yang HJ, Hwang YJ. Comparison of the outcomes of nasal bone reduction using serial imaging. Arch Craniofac Surg 2021;22:193-8.

9. Lee YJ. Orbital floor fracture repair with implants: a retrospective study. Arch Craniofac Surg 2021;22:177-82.

10. Al-Helo S, Alomari RS, Ghosh S, Chaudhary V, Dhillon G, AlZoubi $\mathrm{MB}$, et al. Compression fracture diagnosis in lumbar: a clinical CAD system. Int J Comput Assist Radiol Surg 2013;8: 461-9.

11. Basha CMAKZ, Padmaja M, Balaji GN. Computer aided fracture detection system. J Med Imaging Health Inform 2018;8: 526-31.

12. Chung SW, Han SS, Lee JW, Oh KS, Kim NR, Yoon JP, et al. Automated detection and classification of the proximal humerus fracture by using deep learning algorithm. Acta Orthop 2018;89:468-73.

13. Gale W, Oakden-Rayner L, Carneiro G, Bradley AP, Palmer LJ. Detecting hip fractures with radiologist-level performance using deep neural networks. arXiv 1711.06504 [Preprint]. 2017 [cited 2021 Sep 28]. https://arxiv.org/abs/1711.06504.

14. Kim DH, MacKinnon T. Artificial intelligence in fracture detection: transfer learning from deep convolutional neural networks. Clin Radiol 2018;73:439-45.

15. Lindsey R, Daluiski A, Chopra S, Lachapelle A, Mozer M, Sicular $S$, et al. Deep neural network improves fracture detection by clinicians. Proc Natl Acad Sci U S A 2018;115:11591-6.

16. Olczak J, Fahlberg N, Maki A, Razavian AS, Jilert A, Stark A, et al. Artificial intelligence for analyzing orthopedic trauma radiographs. Acta Orthop 2017;88:581-6.

17. Urakawa T, Tanaka Y, Goto S, Matsuzawa H, Watanabe K, Endo N. Detecting intertrochanteric hip fractures with orthopedist-level accuracy using a deep convolutional neural network. Skeletal Radiol 2019;48:239-44.

18. Yao J, Burns JE, Munoz H, Summers RM. Cortical shell un- 
wrapping for vertebral body abnormality detection on computed tomography. Comput Med Imaging Graph 2014;38:62838.

19. Bayram F, Cakıroglu M. DIFFRACT: DIaphyseal Femur FRActure Classifier SysTem. Biocybern Biomed Eng 2016;36: 157-71.

20. Gulshan V, Peng L, Coram M, Stumpe MC, Wu D, Narayanaswamy A, et al. Development and validation of a deep learning algorithm for detection of diabetic retinopathy in retinal fundus photographs. JAMA 2016;316:2402-10.

21. Esteva A, Kuprel B, Novoa RA, Ko J, Swetter SM, Blau HM, et al. Dermatologist-level classification of skin cancer with deep neural networks. Nature 2017;542:115-8.

22. Nam JG, Park S, Hwang EJ, Lee JH, Jin KN, Lim KY, et al. Development and validation of deep learning-based automatic detection algorithm for malignant pulmonary nodules on chest radiographs. Radiology 2019;290:218-28.

23. Ehteshami Bejnordi B, Veta M, Johannes van Diest P, van Ginneken B, Karssemeijer N, Litjens G, et al. Diagnostic assessment of deep learning algorithms for detection of lymph node metastases in women with breast cancer. JAMA 2017;318: 2199-210.

24. Luo H, Xu G, Li C, He L, Luo L, Wang Z, et al. Real-time artificial intelligence for detection of upper gastrointestinal cancer by endoscopy: a multicentre, case-control, diagnostic study. Lancet Oncol 2019;20:1645-54.

25. Nishimoto S, Sotsuka Y, Kawai K, Ishise H, Kakibuchi M. Personal computer-based cephalometric landmark detection with deep learning, using cephalograms on the internet. J Craniofac Surg 2019;30:91-5.

26. Dhar R, Falcone GJ, Chen Y, Hamzehloo A, Kirsch EP, Noche $\mathrm{RB}$, et al. Deep learning for automated measurement of hemorrhage and perihematomal edema in supratentorial intracerebral hemorrhage. Stroke 2020;51:648-51.

27. Ironside N, Chen CJ, Mutasa S, Sim JL, Ding D, Marfatiah S, et al. Fully automated segmentation algorithm for perihematomal edema volumetry after spontaneous intracerebral hemorrhage. Stroke 2020;51:815-23.

28. Sharrock MF, Mould WA, Ali H, Hildreth M, Awad IA, Hanley $\mathrm{DF}$, et al. 3D deep neural network segmentation of intracerebral hemorrhage: development and validation for clinical trials. Neuroinformatics 2021;19:403-15.

29. Zhao X, Chen K, Wu G, Zhang G, Zhou X, Lv C, et al. Deep learning shows good reliability for automatic segmentation and volume measurement of brain hemorrhage, intraventricular extension, and peripheral edema. Eur Radiol 2021;31:5012-20.

30. Ryu JY, Eo PS, Lee JS, Lee JW, Lee SJ, Lee JM, et al. Surgical approach for venous malformation in the head and neck. Arch
Craniofac Surg 2019;20:304-9.

31. Mundt JC, Vogel AP, Feltner DE, Lenderking WR. Vocal acoustic biomarkers of depression severity and treatment response. Biol Psychiatry 2012;72:580-7.

32. Benba A, Jilbab A, Hammouch A. Discriminating between patients with Parkinson's and neurological diseases using cepstral analysis. IEEE Trans Neural Syst Rehabil Eng 2016;24:1100-8.

33. Lee YW, Bae YC, Park SM, Nam SB, Seo HJ, Kim GW. Outcomes of a superiorly-based pharyngeal flap for the correction of velopharyngeal dysfunction. Arch Craniofac Surg 2020;21: 22-6.

34. Nam SM. Surgical treatment of velopharyngeal insufficiency. Arch Craniofac Surg 2018;19:163-7.

35. Goodfellow IJ, Pouget-Abadie J, Mirza M, Xu B, Warde-Farley D, Ozair S, et al. Generative adversarial nets. In: Ghahramani Z, Welling M, Cortes C, Lawrence N, Weinberger KQ, editors. Advances in neural information processing systems 27. Red Hook: Curran; 2014. p. 2672-80.

36. Zhu JY, Park T, Isola P, Efros AA. Unpaired image-to-image translation using cycle-consistent adversarial networks. Paper presented at: 2017 IEEE International Conference on Computer Vision; 2017 Oct 22-29; Venice, Italy.

37. Mirza M, Osindero S. Conditional generative adversarial nets. arXiv 1411.1784 [Preprint]. 2014 [cited 2021 Sep 28]. https:// arxiv.org/abs/1411.1784.

38. Karras T, Aila T, Laine S, Lehtinen J. Progressive growing of GANs for improved quality, stability, and variation. arXiv 1710.10196 [Preprint]. 2018 [cited 2021 Sep 28]. https://arxiv. org/abs/1710.10196.

39. Wolterink JM, Dinkla AM, Savenije MHF, Seevinck PR, van den Berg CAT, Isgum I. Deep MR to CT synthesis using unpaired data. In: Gooya A, Frangi AF, Tsaftaris SA, Prince JL, editors. Simulation and Synthesis in Medical Imaging. Cham: Springer; 2017. p. 14-23.

40. Hong JY, Han K, Jung JH, Kim JS. Association of exposure to diagnostic low-dose ionizing radiation with risk of cancer among youths in South Korea. JAMA Netw Open 2019;2: e1910584.

41. Karras T, Laine S, Aila T. A style-based generator architecture for generative adversarial networks. IEEE Trans Pattern Anal Mach Intell 2020 Feb 2 [Epub]. https://doi.org/10.1109/TPAMI. 2020.2970919 .

42. Chang HH, Moura JMF. Biomedical signal processing. In: Kutz $\mathrm{M}$, editor, Biomedical engineering and design handbook. Vol. 1. 2nd ed. New York: McGraw-Hill; 2010. p. 559-79.

43. Galloway CD, Valys AV, Shreibati JB, Treiman DL, Petterson FL, Gundotra VP, et al. Development and validation of a deeplearning model to screen for hyperkalemia from the electro- 
cardiogram. JAMA Cardiol 2019;4:428-36.

44. Kwon JM, Jeon KH, Kim HM, Kim MJ, Lim S, Kim KH, et al. Deep-learning-based out-of-hospital cardiac arrest prognostic system to predict clinical outcomes. Resuscitation 2019;139:8491.

45. Suh JM, Chung $\mathrm{CH}$, Chang YJ. Head and neck reconstruction using free flaps: a 30-year medical record review. Arch Craniofac Surg 2021;22:38-44.

46. Marchesi A, Garieri P, Amendola F, Marcelli S, Vaienti L. Intraoperative near-infrared spectroscopy for pedicled perforator flaps: a possible tool for the early detection of vascular issues.
Arch Plast Surg 2021;48:457-61.

47. Salgarello M, Pagliara D, Rossi M, Visconti G, Barone-Adesi L. Postoperative monitoring of free DIEP flap in breast reconstruction with near-infrared spectroscopy: variables affecting the regional oxygen saturation. J Reconstr Microsurg 2018;34: 383-8.

48. Akita S, Mitsukawa N, Tokumoto H, Kubota Y, Kuriyama M, Sasahara Y, et al. Regional oxygen saturation index: a novel criterion for free flap assessment using tissue oximetry. Plast Reconstr Surg 2016;138:510e-518e. 